Portland State University

PDXScholar

Electrical and Computer Engineering Faculty

Publications and Presentations

Electrical and Computer Engineering

6-1-1982

\title{
Thermal Hysteresis in Acoustic Resonators
}

Lee W. Casperson

Portland State University

Lloyd M. Davis

John D. Harvey

Follow this and additional works at: https://pdxscholar.library.pdx.edu/ece_fac

Part of the Electrical and Computer Engineering Commons

Let us know how access to this document benefits you.

Citation Details

Casperson, L. W., Davis, L. M., and Harvey, J. D. (1982). Thermal hysteresis in acoustic resonators. 71 (6), $1412-1416$

This Article is brought to you for free and open access. It has been accepted for inclusion in Electrical and Computer Engineering Faculty Publications and Presentations by an authorized administrator of PDXScholar. Please contact us if we can make this document more accessible: pdxscholar@pdx.edu. 


\title{
Thermal hysteresis in acoustic resonators
}

\author{
Lee W. Casperson, a) Lloyd M. Davis, and John D. Harvey
}

Department of Physics, University of Auckland, Auckland, New Zealand

(Received 21 December 1981; accepted for publication 9 March 1982)

\begin{abstract}
In some applications of piezoelectrically driven acoustic resonators, the power dissipated in the resonating material may be sufficient to significantly change the temperature and hence the resonance frequency. $A$ straightforward analysis shows that this effect can lead to severe hysteresis and thermal bistability. This model is in agreement with experiments that have been performed using a fused silica acousto-optic modulator.
\end{abstract}

PACS numbers: $43.25 . \mathrm{Gf}$, 43.85. Kr, 43.88.Fx

\section{INTRODUCTION}

Acoustic resonance phenomena in solids are finding increasing numbers of practical applications where high $Q$ and frequency stability are important criteria. Well-known examples include crystal filters and frequency standards. In all such systems there are inevitably loss mechanisms, and the dissipation associated with these losses always raises the temperature of the resonator above the ambient temperature. If the device characteristics are functions of temperature, the resonator response will necessarily be a nonlinear function of the excitation amplitude. For most applications this nonlinearity can be avoided by simply maintaining the excitation at a sufficiently low level and keeping the ambient temperature constant. However, for other applications large excitation levels are inevitable. With acousto-optic devices, for example, the efficiency of light diffraction is often roughly proportional to the intensity of the acoustic waves, and there may be practical reasons for operating an acousto-optic modulator at a power level where thermal effects cannot be neglected.

The existence of nonlinearities in acoustic resonance phenomena is well known, and several different types of nonlinearities have been discussed. One of the first and most important nonlinearities to be noted is related to power dependent heating of a resonating material. The vibrations create heat due to internal and external friction, and this heat causes changes in the resonant frequency that depend on the amplitude of vibration. ${ }^{1}$ More specifically the frequency change can arise from temperature dependent changes in the density, dimensions, and elastic coefficients of the material, together with possible temperature dependent effects in the supporting structure. In the development of extremely stable quartz frequency standards, a strain dependence of the elastic coefficients has also been observed. ${ }^{2}$ More recently there have been extensive studies, mostly theoretical, of the elastic, electric, and elastoelectric nonlinearities that are intrinsic to all elastic dielectrics. ${ }^{3-6}$ In these studies, losses and associated temperature changes are generally explicitly excluded, although the potential importance of such effects is sometimes recognized. ${ }^{4}$ We have found that for some acousto-optic applications strong thermal nonlinearities are unavoidable, and at least a qualitative understand-

a)Permanent address: School of Engineering and Applied Science, University of California, Los Angeles, CA 90024. ing of these effects is essential in obtaining optimum performance from acousto-optic modulators. In Sec. I we present an analytical model which accounts for the main features of the observed thermal nonlinearities. Specifically, the model explains the observed features of thermal hysteresis and bistability as well as more complex transient effects resulting from abrupt changes in input power or frequency. Experimental evidence in support of the model is discussed in Sec. II.

\section{THEORY}

Before one could commence a rigorous investigation of thermal effects in acousto-optic modulators it would be necessary to have a detailed understanding of all of the amplitude dependent loss mechanisms involved. There have been several studies of loss processes in crystals and glasses. ${ }^{7}$ The acoustic attenuation coefficients for many acousto-optic materials are now known, and there has also been progress in predicting coefficients for new materials. ${ }^{8.9}$ Unfortunately, there are many other loss mechanisms that would depend on the details of the resonator and driver geometries, and the rate of heat flow between these elements and their environment would also be difficult to predict. Accordingly, we will simply adopt the reasonable assumption that the rate of energy dissipation is proportional to the amplitude squared of the acoustic wave, and the proportionality constant will be left for experimental determination.

The basic steady-state amplitude response function for a high $Q$ acoustic resonator can be characterized by the Lorentzian function

$$
|x|^{2}=\left|x_{0}\right|^{2} /\left\{1+\left[2\left(\nu-\nu_{0}\right) / \Delta \nu\right]^{2}\right\},
$$

where $x_{0}$ is the normalized amplitude of the sinusoidal excitation, $x$ is the amplitude of the response, $\nu$ is the frequency of the excitation, $\nu_{0}$ is the center frequency of the resonance, and $\Delta \nu$ is the full width at half-maximum. This type of response function can be easily derived for the simplest resonator consisting of a mass attached to a damped spring, and it also describes well the high $Q$ response of more general oscillating systems. ${ }^{10}$ For the case of acoustic resonators having many Fabry-Perot-like resonant modes, as in the experiments described below, the Lorentzian resonance approximation is valid as long as the resonances are separated by much more than $\Delta \nu$.

The essence of the nonlinearity of interest here is 
that the resonance frequency $\nu_{0}$ is a function of temperature, while the temperature is itself a function of the amplitude $x$ given in Eq. (1). For small amounts of acoustic heating it is reasonable to suppose that the resonance frequency would be a linear function of the temperature deviation from the ambient temperature $T_{0^{\circ}}$ Therefore Eq. (1) can be rewritten in the form

$$
|x|^{2}=\left|x_{0}\right|^{2} /\left(1+\left\{2\left[\nu-\nu_{0}-a\left(T-T_{0}\right)\right] / \Delta \nu\right\}^{2}\right),
$$

where $a$ is the appropriate temperature coefficient. It is also convenient to introduce a normalized frequency parameter $y=2\left(\nu-\nu_{0}\right) / \Delta \nu$ and a normalized temperature coefficient $\alpha=2 a / \Delta \nu$. Thus Eq. (2) reduces to

$$
|x|^{2}=\left|x_{0}\right|^{2} /\left\{1+\left[y-\alpha\left(T-T_{0}\right)\right]^{2}\right\} .
$$

The temperature of the acoustic resonator is governed by the energy balance equation ${ }^{11}$

$$
\frac{d T}{d t}=-\frac{T-T_{0}}{\tau}+b P,
$$

where $\tau$ is the temperature relaxation time, $P$ is the absorbed power, and the coefficient $b$ is related to the volume and specific heat of the resonating material. It may also be noted that the absorbed power is proportional to the amplitude squared of the oscillation. ${ }^{12}$ Thus if the various coefficients were known, Eqs. (3) and (4) would provide a complete description of the time dependence of the temperature and absorbed power for various values of the frequency and input power.

To examine the implications of Eqs. (3) and (4) it is helpful to introduce the normalized set of variables $T^{\prime}=\alpha\left(T-T_{0}\right), P^{\prime}=\tau \alpha b P, t^{\prime}=t / \tau$. Then Eqs. (3) and (4) can be written simply

$$
\begin{aligned}
& \frac{d T^{\prime}}{d t^{\prime}}=-T^{\prime}+P^{\prime}, \\
& P^{\prime}=P_{0}^{\prime} /\left[1+(y-T)^{2}\right],
\end{aligned}
$$

where $P_{0}^{\prime}$ represents the incident power level. The steady-state solutions are of particular interest. In the limit of very slow variations of $P_{0}^{\prime}$ and $y$ one finds $T^{\prime}=P^{\prime}$ and Eq. (6) is

$$
P^{\prime}=P_{0}^{\prime} /\left[1+(y-P)^{2}\right] \text {. }
$$

This is a cubic equation for $P^{\prime}$, and the solutions may be obtained either numerically or analytically.

The solutions of Eq. (7) are plotted in Fig. 1 for various values of $P_{0}^{\prime}$. The resulting curves look much like the ordinary Lorentzian functions implied by Eq. (1) except that the peaks of the curves are displaced toward higher frequencies. The most striking feature of these results is that for large values of the incident power $P_{0}^{\prime}$ the absorbed power is double valued. Thus the response of an acoustic resonator to an excitation at frequency $y$ depends on the past history of the system. A similar behavior has also been attributed to nonlinear elastic coefficients in quartz. ${ }^{5}$ The basic idea here is that if the excitation frequency is slowly increased from negative values for a fixed $P_{0}^{\prime}$, then the response $P^{\prime}$ will follow up along the top side of the tilted Lorentzian until it falls off the tip of the curve. From there the response will abruptly jump to the lower right-hand wing of the

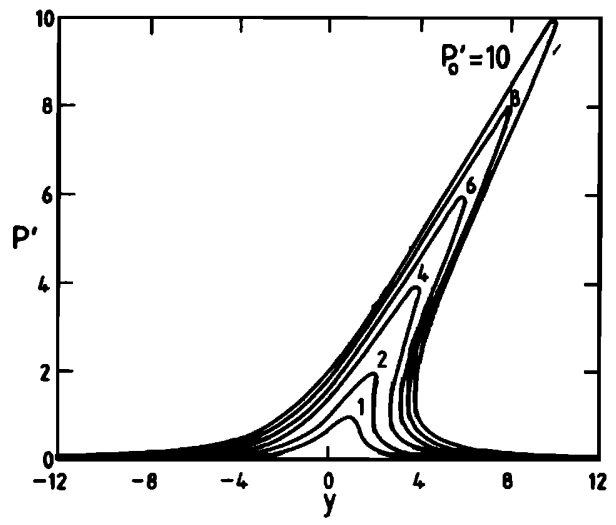

FIG. 1. Normalized absorbed power $P^{\prime}$ as a function of the normalized frequency $y=2\left(\nu-\nu_{0}\right) / \Delta \nu$ for various values of the incident power $P_{0}^{\prime}$.

curve. When the driving frequency is decreased from a high value, on the other hand, the corresponding jump occurs at a lower frequency. As discussed below, the nature of these jumps can be much more complicated if the frequency changes are not slow compared to the thermal relaxation time $\tau$; i.e., if the steady-state approximation ceases to be valid.

Several aspects of the curves shown in Fig. 1 can be described in simple analytical terms. In particular, the maximum response is always $P_{0}^{\prime}$ and this value is obtained at the frequency $y_{\text {peak }}=P_{0}^{\prime}$. The half-power points occur at the frequencies $y_{1 / 2}=P_{0}^{\prime} / 2 \pm 1$. For very small values of $P_{0}^{\prime}$ no bistability occurs, and this effect first appears at $P_{0}^{\prime}=8(27)^{-1 / 2} \simeq 1.54$. For this marginal case the important right-hand inflection point occurs at $y=3^{1 / 2}, P^{\prime}=2(3)^{-1 / 2}$.

Typical time-dependent solutions of Eqs. (5) and (6) are given in Fig. 2 for the situation in which the normalized frequency $y$ is increased in steps of $\Delta y=1$ beginning at large negative values. The horizontal axis corresponds for compactness to both frequency and time, and the interpretation of these curves can be

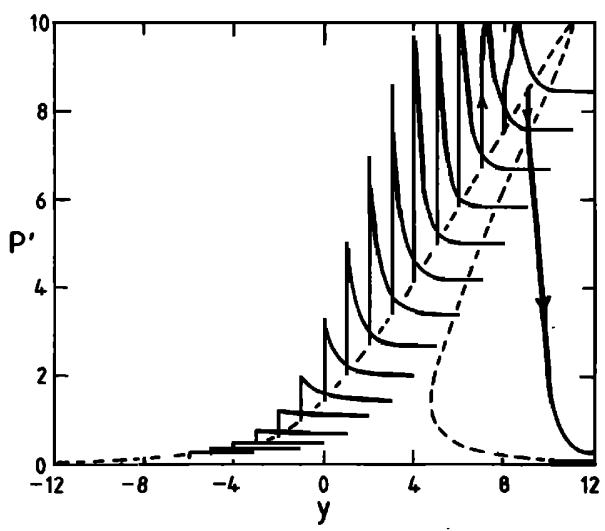

FIG. 2. Absorbed power as a function of frequency and time for $P_{0}^{\prime}=10$ as the frequency is increased in steps of $\Delta y=1$. Thus the curve commencing with a vertical line above $y=0$, for example, represents the time dependence of $P^{\prime}$ when $y$ has just been switched from steady-state operation at $y=-1$. The time scale of the curves is such that a time increment of $\Delta t^{\prime}$ $=1$ corresponds to a $y$ scale translation of $\Delta y=2$. The dashed curve is the steady-state $P_{0}=10$ plot from Fig. 1 translated to the right by $\Delta y=1$. 
illustrated by considering a particular example. Thus one of the curves in Fig. 2 includes a vertical line segment at the frequency $y=-1$ which extends from about $P^{\prime}=1.1$ to $P^{\prime}=2.0$. This is meant to indicate that at some instant of time the resonator is switched from a steadystate value of $P^{\prime}=1.1$ at the frequency $y=-2$ to the new frequency $y=-1$. Immediately after this frequency switch the absorbed power rises instantly to about $P^{\prime}$ $=2.0$. Such an abrupt change is an obvious consequence of Eq. (6) for times so short that $T^{\prime}$ remains constant. As the time $t^{\prime}$ increases the power decays more-or-less exponentially to a new steady-state value of about $P^{\prime}$ $=1.4$, and it is this decaying behavior that is represented by the curved lines in the figure. The time scale for these plots is such that an increase in the normalized time $t^{\prime}$ of the amount $\Delta t^{\prime}=1$ corresponds to a displacement along the $y$ axis of $\Delta y=2$.

The rather complicated looking features of Fig. 2 can all be interpreted physically. For instance when the resonator has been operating for a long time at the frequency $y=4$, the absorbed power will have arrived at the steady-state value $P^{\prime}=5$ as shown in Fig. 1 . If the frequency were from this point scanned around so quickly that the temperature could not vary, the power curve would be found to be an ordinary Lorentzian of maximum value $P^{\prime}=10$, centered at $y=5$, and having its halfpower point, where the discussion started-at $y=4, P^{\prime}$ $=5$. Therefore, as Fig. 2 reveals, when the frequency is abruptly switched from $y=4$ to $y=5$ the absorbed power jumps to $P^{\prime}=10$. If the frequency is left at this new value, additional heating occurs and the center frequency of the ordinary Lorentzian described above slowly slides to a higher frequency causing a gradual reduction in the absorbed power. If the frequency is then increased to a higher value, the gradual heating causes the peak of the ordinary Lorentzian to scan past the applied frequency. Thus the curves starting to the right of $y=5$ have rounded tops peaking at $P^{\prime}=10$. Eventually a point is reached where a jump upward in frequency (to $y=9$ in this case) requires an instantaneous decrease in absorbed power. The resulting decline in temperature causes the ordinary Lorentzian to shift left which leads to further decreases in absorption. This process continues until a steady operating point is reached on the right-hand wing of the Lorentzian. The dashed lines in the figure are intended to represent the fact that the steady-state operating points fall on the $P_{0}^{\prime}=10$ curve of Fig. 1 (in this representation the curve is shifted to the right by $\Delta t^{\prime}=0.5$ or $\Delta y=1$ ).

Solutions of Eqs. (5) and (6) are given in Fig. 3 for the case in which the normalized frequency $y$ is decreased in steps of $\Delta y=1$ beginning at large positive values. The interpretation of these curves is essentially the same as in the previous case and little comment is needed here. Again both time and frequency increase to the right. Perhaps the most interesting single phenomenon in Fig. 3 occurs after the frequency is reduced from $y=4$ to $y=3$. At that time there is a brief absorption peak as the center frequency of the ordinary Lorentzian slides past $y=3$. From a comparison of Figs. 2 and 3 it is clear that high absorption levels are only possible if the resonance frequency is approached from be-

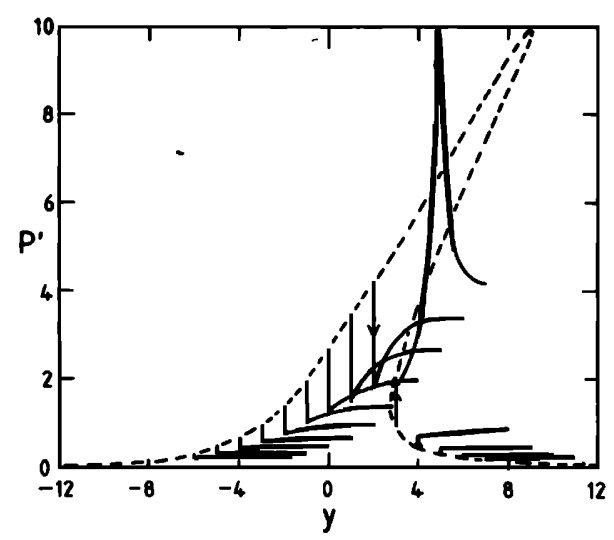

FIG. 3. Absorbed power as a function of frequency and time for $P_{0}^{\prime}=10$ as the frequency is decreased in steps of $\Delta y=1$. Interpretation of these plots is as in Fig. 2, and the dashed curve is the $P_{0}^{\prime}=10$ plot from Fig. 1 shifted to the left by $\Delta y$ $=1$.

low. Even when this is done the frequency steps (or the rate of change of frequency with time) must be kept very small to achieve anywhere close to the maximum value of $P^{\prime}=P_{0^{\circ}}^{\prime}$ In Fig. 2, for example, the frequency steps were $\Delta y=1$ and the highest steady-state power achieved was about $0.84 P_{0^{\circ}}^{\prime}$

\section{EXPERIMENT}

We have performed several experiments on an acoustic resonator which exhibits the hysteresis phenomena discussed in the previous section. The resonator is a fused silica prism which is intended for acousto-optical loss modulation of an argon ion laser. ${ }^{13} \mathrm{~A}$ transducer is mounted on the end of the triangular prism, which is approximately $0.55 \mathrm{~cm}$ long $\times 1.15 \times 1.15 \times 0.90 \mathrm{~cm}$. It is kept in a temperature controlled housing to stabilize the ambient temperature, which is monitored with a thermistor. The transducer is driven by a variable frequency RF power supply. The RF power not absorbed by the resonator is reflected back to a directional

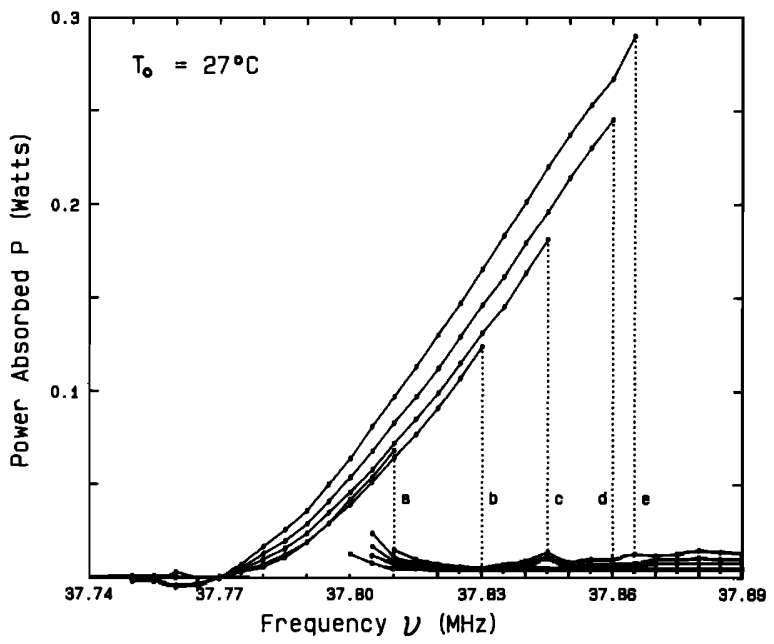

FIG. 4. Experimental plots of steady-state absorbed power versus frequency for various levels of incident power: $a, P_{0}^{\prime}$ $=0.095 \mathrm{~W} ; \mathrm{b}, P_{0}^{\prime}=0.154 \mathrm{~W} ; \mathrm{c}, P_{0}^{\prime}=0.226 \mathrm{~W} ; \mathrm{d}, P_{0}^{\prime}=0.280 \mathrm{~W}$; e, $P_{0}^{\prime}=0.332 \mathrm{~W}$. The ambient temperature remains constant throughout at $27.0^{\circ} \mathrm{C}$. 


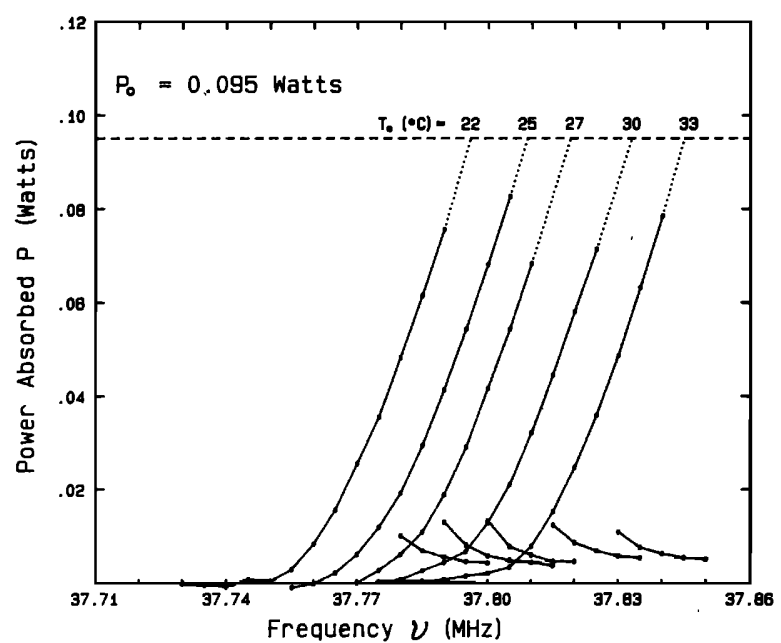

FIG. 5. Experimental plots of steady-state absorbed power versus frequency for various ambient temperatures. The acoustic resonator is driven at an incident power of $0.095 \mathrm{~W}$, and the temperature is measured with a calibrated thermistor.

coupler at the power supply. This reflected RF power is monitored and used to calculate the power absorbed by the resonator.

Plots of absorbed power versus frequency for one resonance of the acousto-optic prism are shown in Fig. 4 for several different values of the incident power. In obtaining these plots the frequency was slowly raised or lowered in very small frequency steps so that as much as possible of the steady-state hysteresis curves could be discerned. The results are seen to be qualitatively identical to the predictions of the theoretical model that has been discussed. The slight discrepancies in the baseline and spacing of the curves are due to power calibration errors and possibly a temperature dependence of the coefficients in Eqs. (4). Extremely strong hysteresis and bistability effects are observed, and the absorption level at a particular frequency may change by more than an order of magnitude depending on the past history of the resonator.

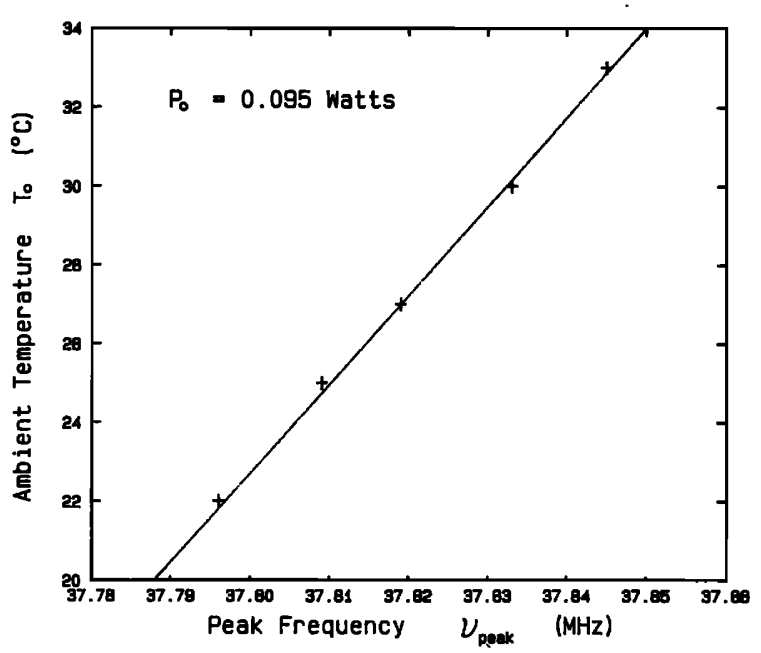

FIG. 6. The frequency of the peaks of the resonances of Fig. 5 versus temperature. This shows that the resonant frequency is linearly dependent on temperature with a coefficient of $a$ $=5.4 \mathrm{kHz}^{\circ} \mathrm{C}^{-1}$.

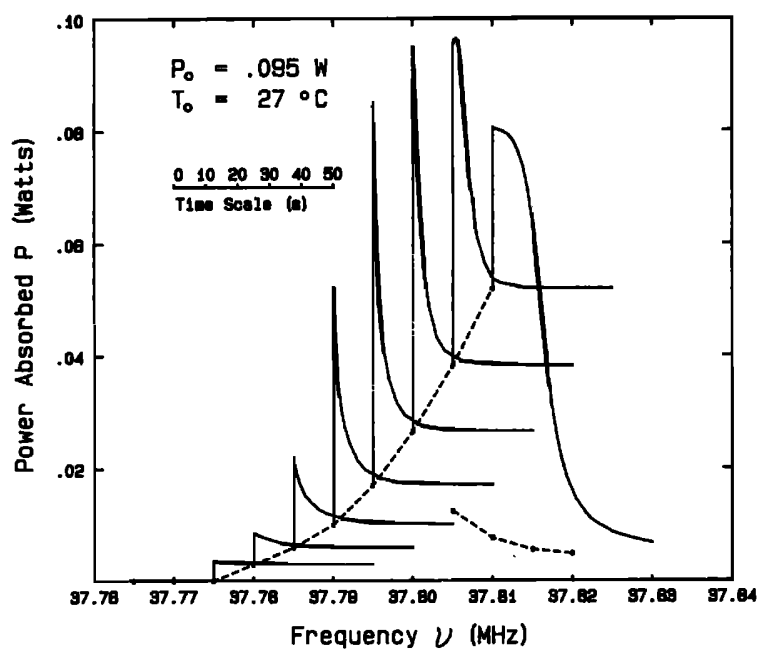

FIG. 7. Absorbed power as a function of time for an incident power of $P_{0}=0.095 \mathrm{~W}$ as the frequency is increased in steps of $\Delta \nu=5 \mathrm{kHz}$. The time dependence is shown for $50 \mathrm{~s}$ in each case. When the frequency is switched from 37805 to 37810 $\mathrm{kHz}$ the absorbed power decreases as the resonator falls out of resonance. Compare with Fig. 2.

To demonstrate that the effects are thermal in nature, the resonance is plotted out in Fig. 5 at different ambient temperatures for the same incident power. From this figure it is clear that small temperature changes can have a significant effect on the frequency characteristics. Figure 6 is obtained by plotting the positions of the extrapolated peaks in Fig. 5 as a function of temperature. The temperature dependence is found to be linear in agreement with Eq. (2), with an experimental temperature coefficient of $a=5.4 \mathrm{kHz}^{\circ} \mathrm{C}^{-1}$. Assuming a resonance width of $\Delta \nu=4 \mathrm{kHz}$ we obtain an experimental estimate for the coefficient $\alpha$ of $2.7^{\circ} \mathrm{C}^{-1}$.

To test the more detailed predictions of the time-dependent model, we have altered the excitation frequency in steps of $5 \mathrm{kHz}$ and noted the absorbed power as a function of time. The results as displayed in Figs. 7

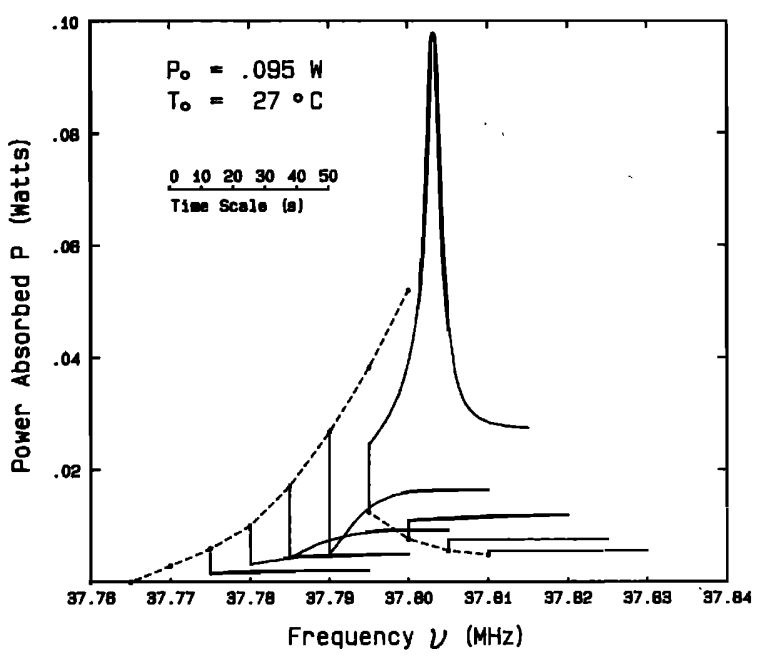

FIG. 8. As for Fig. 7 except that the frequency is decreased in steps. When the frequency is switched from 37800 to 37795 $\mathrm{kHz}$ the power absorbed increases to a maximum before settling down to a steady level. Compare with Fig. 3. 
and 8 are seen to be in excellent qualitative agreement with the theoretical predictions illustrated in Figs. 2 and 3. From the time dependences shown in these plots it can be inferred that the time constant of Eq. (4) is approximately $\tau=2.5 \mathrm{~s}$. Using the previously noted fact that the peak of the resonance occurs at the normalized frequency $y_{\text {pcak }}=P_{0}^{\prime}$ one can easily show that $\nu_{\text {peak }}-\nu_{0}$ $=a b P_{0} \tau$. Hence the coefficient $b$ can be found for our system to be $23.4{ }^{\circ} \mathrm{C} \mathrm{W}^{-1} \mathrm{~s}^{-1}$. All of the coefficients appearing in Eqs. (2) and (3) have now been determined, so that the response of our system could be predicted theoretically for arbitrary time-dependent operating conditions.

\section{CONCLUSION}

For some applications of acoustic resonators the power levels are sufficient to cause a slight heating of the resonating material. This heating leads to changes in the elastic properties and dimensions of the resonator and its supporting structure, and inevitably there are also changes in the resonance frequency. In a high $Q$ system these changes may be sufficient to introduce severe hysteresis in the device's operating characteristics. In the present study the implications of this thermally induced hysteresis have been examined in detail. An analytical model has been developed in which the center frequency of a Lorentzian absorption resonance varies linearly with temperature, while the temperature obeys a relaxation equation driven by the absorbed power. This model is found to account well for all of the rather complex features of our experimental data involving an acousto-optic modulator. A practical consequence of the model is that obtaining optimum performance from a thermally nonlinear acoustic resonator will require close attention to the rate and direction in which frequency and power changes are made. By use of the model described here it should be possible to undertake such optimization in a systematic manner. Also, it would seem that the strong thermal nonlinearit- ies described here might often dominate the more subtle Hooke's law nonlinearities that have received such extensive theoretical treatment. Consequently, in any experimental demonstration of nonlinear elastic coefficients considerable care may be required to ensure that thermal effects are not also occurring.

\section{ACKNOWLEDGMENT}

The authors are pleased to acknowledge that this work was supported in part by a grant from The Cancer Society of New Zealand (Auckland Division).

${ }^{1}$ E. A. Gerber, Electronics 24, 142 (April 1951); and 326 (September 1951).

${ }^{2}$ A. W. Warner, Bell. Syst. Tech. J. 39,1193 (1960), Sec. 4.3. ${ }^{3}$ R. N. Thurston and K. Brugger, Phys. Rev. 133A, 1611 (1964).

${ }^{4}$ R. N. Thurston and M. J. Shapiro, J. Acoust. Soc. Am. 41, 1112 (1967).

${ }^{5}$ J. J. Gagnepain and R. Besson, Physical Acoustics, Vol. XI, edited by W. P. Mason and R. N. Thurston (Academic, New York, 1975), p. 245.

${ }^{6}$ D. F. Nelson, J. Acoust. Soc. Am . 63, 1738 (1978), and references therein.

${ }^{7}$ W. P. Mason, Physical Acoustics and the Properties of Solids (Van Nostrand, Princeton, 1958), Chap. 10 and references therein.

${ }^{8} \mathrm{M}$. G. Holland, IEEE Trans. Sonics Ultrason. SU-15, 18 (1968).

${ }^{9}$ D. A. Pinnow, IEEE J. Quant. Electron. QE-6, 223 (1970). ${ }^{10} \mathrm{~A}$. Yariv, Introduction to Optical Electronics (Holt, New York, 1976), p. 91.

${ }^{11}$ R. A. Smith, F. E. Jones, and R. P. Chasmar, The Detection and Measurement of Infra-Red Radiation (Oxford, London, 1968), p. 50.

${ }^{12}$ P. M. Morse, Vibration and Sound (McGraw-Hill, New York, 1948), Sec. II.4.

${ }^{13}$ Coherent Radiation Model 467 mode locker. 